\title{
A Note on Control of A Class of Discrete-Time Stochastic Systems with Distributed Delays and Nonlinear Disturbances *
}

\author{
Zidong Wang a,b, Yurong Liu ${ }^{\mathrm{c}}$, Guoliang Wei ${ }^{\mathrm{b}}$, Xiaohui Liu ${ }^{\mathrm{a}}$ \\ ${ }^{a}$ Department of Information Systems and Computing, Brunel University, Uxbridge, Middlesex, UB8 3PH, U.K. \\ ${ }^{\mathrm{b}}$ School of Information Science and Technology, Donghua University, Shanghai 200051, China. \\ ${ }^{\mathrm{c}}$ Department of Mathematics, Yangzhou University, Yangzhou 225002, P. R. China.
}

\begin{abstract}
This paper is concerned with the state feedback control problem for a class of discrete-time stochastic systems involving sector nonlinearities and mixed time delays. The mixed time-delays comprise both the discrete and distributed delays, and the sector nonlinearities appear in the system states and all delayed states. The distributed time-delays in the discrete-time domain are first defined and then a special matrix inequality is developed to handle the distributed time-delays within an algebraic framework. An effective linear matrix inequality (LMI) approach is proposed to design the state feedback controllers such that, for all admissible nonlinearities and time-delays, the overall closed-loop system is asymptotically stable in the mean square sense. Sufficient conditions are established for the nonlinear stochastic time-delay systems to be asymptotically stable in the mean square sense, and then the explicit expression of the desired controller gains is derived. A numerical example is provided to show the usefulness and effectiveness of the proposed design method.
\end{abstract}

Key words: Discrete-time nonlinear stochastic system; mixed time delays; Lyapunov-Krasovskii functional; linear matrix inequality.

\section{Introduction}

In the past few decades, stochastic dynamical systems modeled by the Itô-type stochastic differential or difference equations have received a great deal of research attention since stochastic systems have many applications in practice such as attitude control of satellites and missile autopilot control, macroeconomic system control and chemical process control $[14,19]$. Although a variety of results for the stability and stabilization of linear stochastic systems have been published, the stabilization control problem of nonlinear stochastic systems has received relatively little attention.

Recently, several important results have been obtained in the area of nonlinear stochastic control, see e.g. [2, $8,16]$. In particular, the so-called sector nonlinearity [9] has gained much attention for deterministic systems, and both the control analysis and model reduction problems have been investigated, see $[7,12]$. On the other hand, stability analysis of time-delay systems has been a problem of recurring interest during the past years [1,15]. For linear stochastic time-delay systems, the stability and stabilization problems have also been studied by many

\footnotetext{
* This work was supported in part by the Engineering and Physical Sciences Research Council (EPSRC) of the U.K. under Grant GR/S27658/01, the Royal Society of the U.K., the National Natural Science Foundation of China under Grants 60774073 and 60974030, the National 973 Program of China under Grant 2009CB320600, and the Alexander von Humboldt Foundation of Germany. Corresponding author Guoliang Wei.
} Wei).

Email address: Guoliang.Wei1973@gmai.com (Guoliang authors, see e.g. [5, 18]. It should be noticed that almost all time-delays studied in the aforementioned literature are of the discrete nature. Recently, another type of time-delays, namely, distributed time delays, has recently drawn much research interests. This is mainly because the signal propagation is often distributed during a certain time period with the presence of an amount of parallel pathways with a variety of axon sizes and lengths $[3,4]$. It is worth mentioning that, the general method of Lyapunov functionals construction has been proposed in [11] and the stability results have been established in [17] for difference equations with distributed and varying delays. In fact, both discrete and distributed delays should be taken into account when modeling a realistic complex systems, and it is not surprising that various systems with discrete and distributed delays (also called mixed delays) have drawn increasing research attention, see $[13,20]$ and the references cited therein.

Although the importance of distributed delays has been widely recognized, almost all available results have been focused on continuous-time systems with distributed delays that are described in the form of a finite or infinite integral. In reality, however, discrete-time systems become more important than their continuous-time counterparts when implementing the control laws in a digital way. To be more specific, it is essential to formulate discretetime analogue of the continuous-time system when one wants to simulate or compute the continuous-time one after obtaining its dynamical characteristics. Naturally, it turns out to be meaningful to investigate the issue of how distributed delays influence the dynamical behavior of a discrete-time system. Unfortunately, a literature search has revealed that such an issue has not yet been 
addressed, and the main reason lies in how to properly define the distributed delays in a discrete-time domain and how to carry out the corresponding mathematical analysis. It is, therefore, the purpose of this paper to close such a gap by making one of the first few attempts to deal with the control problem for a class of discrete-time nonlinear stochastic systems with distributed delays.

Notations: Throughout this paper, $\mathbb{N}^{+}$stands for the set of nonnegative integers; $\mathbb{R}^{n}$ and $\mathbb{R}^{n \times m}$ denote, respectively, the $n$ dimensional Euclidean space and the set of all $n \times m$ real matrices. The superscript " $T$ " denotes the transpose and the notation $X \geq Y$ (respectively, $X>Y$ ) where $X$ and $Y$ are symmetric matrices, means that $X-Y$ is positive semi-definite (respectively, positive definite). $I$ is the identity matrix with compatible dimension. $|\cdot|$ denotes the Euclidean norm in $\mathbb{R}^{n}$. If $A$ is a symmetric matrix, $\lambda_{\max }(A)$ (respectively, $\lambda_{\min }(A)$ ) denote the largest (respectively, smallest) eigenvalue of $A$. Moreover, we may fix a probability space $(\Omega, \mathcal{F}, \mathcal{P})$ where, $\mathcal{P}$, the probability measure, has total mass $1 . \mathbb{E}\{\cdot\}$ stands for the mathematical expectation operator with respect to the given probability measure $\mathcal{P}$. The asterisk $*$ in a matrix is used to denote term that is induced by symmetry. Matrices, if not explicitly specified, are assumed to have compatible dimensions. Sometimes, the arguments of a function will be omitted in the analysis when no confusion can arise.

\section{Problem Formulation}

Consider, on a probability space $(\Omega, \mathcal{F}, \mathcal{P})$, the following discrete-time nonlinear stochastic system with mixed time delays of the form:

$$
\begin{aligned}
(\Sigma): x(k+1)= & A x(k)+B x(k-d(k)) \\
& +C \sum_{m=1}^{+\infty} \mu_{m} f(x(k-m)) \\
& +g(x(k), x(k-d(k)))+D u(k) \\
& +\sigma(x(k), x(k-d(k))) w(k), \\
x(j)= & \phi(j), j=-d_{M},-d_{M}+1, \ldots,-1,0,
\end{aligned}
$$

where $x(k) \in \mathbb{R}^{n_{x}}$ is the state vector; $u(k) \in \mathbb{R}^{n_{u}}$ is the control input; $A, B, C$, and $D$ are known constant matrices; $f(\cdot): \mathbb{R}^{n_{x}} \rightarrow \mathbb{R}^{n_{x}}, g(\cdot, \cdot): \mathbb{R}^{n_{x}} \times \mathbb{R}^{n_{x}} \rightarrow \mathbb{R}^{n_{x}}$ and $\sigma(\cdot, \cdot): \mathbb{R}^{n_{x}} \times \mathbb{R}^{n_{x}} \rightarrow \mathbb{R}^{n_{x}}$ are nonlinear functions; $w(k)$ is a scalar Wiener process (Brownian Motion) defined on a complete probability space $(\Omega, \mathcal{F}, \mathcal{P})$ with

$$
\mathbb{E}[w(k)]=0, \mathbb{E}\left[w^{2}(k)\right]=1, \mathbb{E}\{w(i) w(j)\}=0(i \neq j)
$$

where the stochastic variables $w(0), w(1), w(2), \ldots$ are assumed to be mutually independent; $\phi(j), j=$ $-d_{M},-d_{M}+1, \ldots,-1,0$, are the initial conditions, which are independent of the process $\{w(\cdot)\}$.

In the system $(\Sigma)$, the positive integer $d(k)$ denotes the time-varying delay satisfying

$d_{m} \leq d(k) \leq d_{M}, k \in \mathbb{N}^{+}$

where $d_{m}$ and $d_{M}$ are known positive integers. The constants $\mu_{m} \geq 0(m=1,2, \ldots)$ satisfies the following convergence conditions:

$$
\sum_{m=1}^{+\infty} \mu_{m}<+\infty \text { and } \sum_{m=1}^{+\infty} m \mu_{m}<+\infty \text {. }
$$

Remark 1 The model (1) includes the term of the distributed time-delays, $\sum_{m=1}^{+\infty} \mu_{m} f(x(k-m))$, in the discrete-time setting. Such a term can be interpreted as the discrete analogy of the following continuous-time system with mixed time delay (see e.g. [13]):

$$
\begin{aligned}
d x(t)= & {[A x(t)+B x(t-\tau(t))+g(x(t), x(t-d(t)))} \\
& \left.+C \int_{-\infty}^{t} k(t-s) f\left(x_{i}(s)\right) d s+D u(t)\right] d t \\
& +\sigma(x(t), x(t-d(t))) d w(t) .
\end{aligned}
$$

As can be seen later, the inclusion of such a distributed delay term will bring additional difficulty in the analysis and a special inequality will need to be developed.

For the nonlinear vector functions $f, g$ and $\sigma$, we assume:

$$
\begin{aligned}
& {\left[f(x)-L_{1} x\right]^{T}\left[f(x)-L_{2} x\right] \leq 0, \forall x \in \mathbb{R}^{n_{x}},} \\
& |\sigma(x, y, t)|^{2} \leq\left|\Sigma_{1} x\right|^{2}+\left|\Sigma_{2} y\right|^{2}, \forall x, y \in \mathbb{R}^{n_{x}}, \\
& |g(x, y, t)|^{2} \leq\left|G_{1} x\right|^{2}+\left|G_{2} y\right|^{2}, \forall x, y \in \mathbb{R}^{n_{x}},
\end{aligned}
$$

where $L_{1}, L_{2}, \Sigma_{1}, \Sigma_{2}, G_{1}, G_{2} \in \mathbb{R}^{n_{x} \times n_{x}}$ are known real constant matrices, and $\rho_{1}$ and $\rho_{2}$ are known real scalar constants.

Remark 2 Note that the nonlinear vector functions $\sigma$ and $g$ satisfy the norm-bounded conditions, and $f$ satisfies the so-called sector condition in the sense that $f$ belongs to the sector $\left[L_{1}, L_{2}\right][9]$. Such a sector description is quite general that includes the usual Lipschitz conditions as a special case, and also covers several other classes of well-studied nonlinear systems [7, 12].

Substituting the state feedback controller $u(k)=K x(k)$ to system $(\Sigma)$ gives the following closed-loop system:

$$
\begin{aligned}
\left(\Sigma_{c}\right): x(k+1)= & A_{K} x(k)+B x(k-d(k)) \\
& +C \sum_{m=1}^{+\infty} \mu_{m} f(x(k-m)) \\
& +g(x(k), x(k-d(k))) \\
& +\sigma(x(k), x(k-d(k))) w(k), \\
x(k)= & \phi(k),-\infty<j \leq 0,
\end{aligned}
$$

where $A_{K}=A+D K$.

Definition 1 The system $(\Sigma)$ with $u(k) \equiv 0$ is said to be asymptotically stable in the mean square sense if, there exists a constant $R_{0}>0$ such that for any initial condition $\left\{\phi(j) ;|\phi(j)| \leq R_{0},-\infty<j \leq 0\right\}$, the corresponding solution $\{x(k) ; k \geq 1\}$ satisfies $\lim _{k \rightarrow \infty} \mathbb{E}\left[|x(k)|^{2}\right]=0$.

Definition 2 The system $(\Sigma)$ with $u(k) \equiv 0$ is said to be globally asymptotically stable in the mean square sense if, for any initial condition, the corresponding solution $\{x(k) ; k \geq 1\}$ satisfies $\lim _{k \rightarrow \infty} \mathbb{E}\left[|x(k)|^{2}\right]=0$.

Definition 3 The system $(\Sigma)$ is said to be stabilizable in the mean square sense if there exists a state feedback controller $u(t)=K x(t)$ such that the close-loop $\left(\Sigma_{c}\right)$ is asymptotically stable in the mean square sense. 
In this paper, we aim at developing techniques for stochastically stabilizing a class of discrete-time nonlinear stochastic systems $(\Sigma)$ with mixed time delays. By constructing new Lyapunov-Krasovskii functional, we shall establish LMI-based sufficient conditions under which the stabilizability in mean square sense is guaranteed for the stochastic system $(\Sigma)$.

\section{Main Results}

The following lemmas are essential in establishing our main results.

Lemma 1 [13] Let $x, y$ be any $n_{x}$-dimensional real vectors, and let $P$ be a $n_{x} \times n_{x}$ positive semi-definite matrix. Then, we have $2 x^{T} P y \leq x^{T} P x+y^{T} P y$.

Lemma 2 [13] Let $M \in \mathbb{R}^{n_{x} \times n_{x}}$ be a positive semidefinite matrix, $\boldsymbol{x}_{i} \in \mathbb{R}^{n_{x}}$ and $a_{i} \geq 0(i=1,2, \ldots)$. If the series concerned is convergent, then the following inequality holds:

$$
\left(\sum_{i=1}^{+\infty} a_{i} \boldsymbol{x}_{i}\right)^{T} M\left(\sum_{i=1}^{+\infty} a_{i} \boldsymbol{x}_{i}\right) \leq\left(\sum_{i=1}^{+\infty} a_{i}\right) \sum_{i=1}^{+\infty} a_{i} \boldsymbol{x}_{i}^{T} M \boldsymbol{x}_{i}
$$

For notation simplicity, we denote

$$
\begin{aligned}
& \mathcal{H}(k)=A_{K} x(k)+B x(k-d(k))+g(x(k), x(k-d(k))) \\
& +C \sum_{m=1}^{+\infty} \mu_{m} f(x(k-m)) \text {, } \\
& \xi(k)=\left[x^{T}(k) x^{T}(k-d(k)) f^{T}(x(k))\right. \\
& \left.\sum_{m=1}^{+\infty} \mu_{m} f^{T}(x(k-m)) g^{T}(x(k), x(k-d(k)))\right]^{T}, \\
& \xi_{0}(k)=\left[\begin{array}{llll}
x^{T}(k) & x^{T}(k-d(k)) & x^{T}\left(k-d_{M}\right) & f^{T}(x(k))
\end{array}\right. \\
& \left.\sum_{m=1}^{+\infty} \mu_{m} f^{T}(x(k-m)) g^{T}(x(k), x(k-d(k)))\right]^{T}, \\
& \eta=\left[\begin{array}{lllll}
A_{K} & B & 0 & C & I
\end{array}\right], \bar{\mu}=\sum_{m=1}^{+\infty} \mu_{m}, \\
& \breve{L}_{1}=\left(L_{1}^{T} L_{2}+L_{2}^{T} L_{1}\right) / 2, \breve{L}_{2}=\left(L_{1}^{T}+L_{2}^{T}\right) / 2, \\
& \eta_{0}=\left[\begin{array}{llllll}
A_{K} & B & 0 & 0 & C & I
\end{array}\right] \text {. }
\end{aligned}
$$

Theorem 1 Let $K$ be a given real constant matrix. The closed-loop system $\left(\Sigma_{c}\right)$ is globally asymptotically stable in the mean square sense if there exist six positive definite matrices $X, Q, S, R, Z_{1}$ and $Z_{2}$, a positive constant scalar $\lambda$, and three matrices $M_{1}, M_{2}$ and $M_{3}$ such that the following LMIs hold:

$$
\Psi=\left[\begin{array}{cc} 
& P<\lambda I \\
\Psi_{0}+\Psi_{1}+\Xi_{1}+\Xi_{1}^{T}+\Xi_{2}+\Xi_{2}^{T} & \Xi_{3} \\
\Xi_{3}^{T} & \Xi_{4}
\end{array}\right]<0 .
$$

where

$$
\begin{aligned}
& \Psi_{1}=\eta_{0}^{T}\left(P+d_{M}\left(Z_{1}+Z_{2}\right)\right) \eta_{0},
\end{aligned}
$$

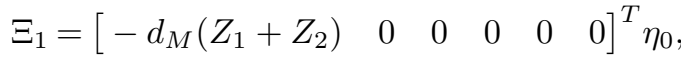

$$
\begin{aligned}
& \Xi_{2}=\left[\begin{array}{llllll}
M_{1}+M_{3} & M_{2}-M_{1} & -M_{2}-M_{3} & 0 & 0 & 0
\end{array}\right] \text {, } \\
& \Xi_{3}=\left[\begin{array}{lll}
\sqrt{d_{M}} M_{1} & \sqrt{d_{M}-d_{m}} M_{2} & \sqrt{d_{M}} M_{3}
\end{array}\right], \\
& \Xi_{4}=\operatorname{diag}\left\{-Z_{1},-Z_{1},-Z_{2}\right\} \text {, }
\end{aligned}
$$$$
\Psi_{0}=\left[\begin{array}{cccccc}
\Pi_{1} & 0 & 0 & \breve{L}_{2} & 0 & 0 \\
* & \Pi_{2} & 0 & 0 & 0 & 0 \\
* & * & -S & 0 & 0 & 0 \\
* & * & * & \bar{\mu} R-I & 0 & 0 \\
* & * & * & * & -\frac{1}{\bar{\mu}} R & 0 \\
* & * & * & * & * & -I
\end{array}\right],
$$

with

$$
\begin{aligned}
\Pi_{1}= & -P+\left(d_{M}-d_{m}+1\right) Q+\lambda \Sigma_{1}^{T} \Sigma_{1}+S-\breve{L}_{1} \\
& +d_{M}\left(Z_{1}+Z_{2}\right)+G_{1}^{T} G_{1}, \\
\Pi_{2}= & -Q+\lambda \Sigma_{2}^{T} \Sigma_{2}+G_{2}^{T} G_{2} .
\end{aligned}
$$

Proof: To deal with the stability problem of the system $\left(\Sigma_{c}\right)$, we construct the Lyapunov-Krasovskii functional $V(k)=\sum_{i=1}^{6} V_{i}(k)$, where

$$
\begin{aligned}
V_{1}(k)= & x^{T}(k) P x(k), V_{2}(k)=\sum_{i=k-d(k)}^{k-1} x^{T}(i) Q x(i), \\
V_{3}(k)= & \sum_{j=k-d_{M}+1}^{k-d_{m}} \sum_{i=j}^{k-1} x^{T}(i) Q x(i), \\
V_{4}(k)= & \sum_{i=k-d_{M}}^{k-1} x^{T}(i) S x(i), \\
V_{5}(k)= & \sum_{i=-d_{M}}^{-1} \sum_{j=k+i}^{k-1} y^{T}(j)\left(Z_{1}+Z_{2}\right) y(j) \\
& \text { with } y(j)=x(j+1)-x(j), \\
V_{6}(k)= & \sum_{i=1}^{+\infty} \mu_{i} \sum_{j=k-i}^{k-1} f^{T}(x(j)) R f(x(j)) .
\end{aligned}
$$

Calculating the difference of $V(k)$ along the system $\left(\Sigma_{c}\right)$ and taking the mathematical expectation, we have

$$
\begin{aligned}
\mathbb{E}\{\Delta V(k)\} & =\sum_{i=1}^{6} \mathbb{E}\left\{\Delta V_{i}(k)\right\} \\
& =\sum_{i=1}^{6} \mathbb{E}\left\{V_{i}(k+1)-V_{i}(k)\right\},
\end{aligned}
$$

where

$$
\begin{aligned}
\mathbb{E}\{\Delta & \left.V_{1}(k)\right\} \\
=\mathbb{E}\{ & \mathcal{H}^{T}(k) P \mathcal{H}(k)+\sigma^{T}(x(k), x(k-d(k))) \\
& \times P \sigma(x(k), x(k-d(k)))\}-x^{T}(k) P x(k), \\
\mathbb{E}\left\{\Delta V_{2}(k)\right\} & \\
\leq & \mathbb{E}\left\{x^{T}(k) Q x(k)-x^{T}(k-d(k)) Q x(k-d(k))\right.
\end{aligned}
$$




$$
\left.+\sum_{i=k-d_{M}+1}^{k-d_{m}} x^{T}(i) Q x(i)\right\},
$$

$\mathbb{E}\left\{\Delta V_{3}(k)\right\}$

$$
\begin{aligned}
=\mathbb{E}\{ & \left(d_{M}-d_{m}\right) x^{T}(k) Q x(k) \\
& \left.-\sum_{i=k-d_{M}+1}^{k-d_{m}} x^{T}(i) Q x(i)\right\},
\end{aligned}
$$

\section{$\mathbb{E}\left\{\Delta V_{4}(k)\right\}$}

$$
=\mathbb{E}\left\{x^{T}(k) S x(k)-x^{T}\left(k-d_{M}\right) S x\left(k-d_{M}\right)\right\},
$$$$
\mathbb{E}\left\{\Delta V_{5}(k)\right\}
$$

$$
=\mathbb{E}\left\{\left[d_{M} \mathcal{H}^{T}(k)-2 d_{M} x^{T}(k)\right]\left(Z_{1}+Z_{2}\right) \mathcal{H}(k)\right.
$$

$$
\begin{aligned}
& +d_{M} x^{T}(k) Z_{1} x(k)-\sum_{i=k-d(k)}^{k-1} y^{T}(i) Z_{1} y(i) \\
& +d_{M} x^{T}(k) Z_{2} x(k)-\sum_{i=k-d_{M}}^{k-d(k)-1} y^{T}(i) Z_{1} y(i) \\
& \left.-\sum_{i=k-d_{M}}^{k-1} y^{T}(i) Z_{2} y(i)\right\}
\end{aligned}
$$

and

$$
\begin{aligned}
\mathbb{E}\left\{\Delta V_{6}(k)\right\} & \\
=\mathbb{E} & \left\{\bar{\mu} f^{T}(x(k)) R f(x(k))\right. \\
& \left.\quad-\sum_{i=1}^{+\infty} \mu_{i} f^{T}(x(k-i)) R f(x(k-i))\right\} .
\end{aligned}
$$

Substituting (22)-(26) into (20) leads to

$$
\begin{aligned}
& \mathbb{E}\{\Delta V(k)\} \\
& \leq \mathbb{E}\left\{\mathcal{H}^{T}(k) P \mathcal{H}(k)-x^{T}(k-d(k)) Q x(k-d(k))\right. \\
&+\sigma^{T}(x(k), x(k-d(k)) P \sigma(x(k), x(k-d(k))) \\
&+x^{T}(k)\left[-P+\left(d_{M}-d_{m}+1\right) Q\right] x(k) \\
&+x^{T}(k) S x(k)-x^{T}\left(k-d_{M}\right) S x\left(k-d_{M}\right) \\
&+\left[d_{M} \mathcal{H}^{T}(k)-2 d_{M} x^{T}(k)\right]\left(Z_{1}+Z_{2}\right) \mathcal{H}(k) \\
&+d_{M} x^{T}(k)\left(Z_{1}+Z_{2}\right) x(k) \\
&-\sum_{i=k-d(k)}^{k-1} y^{T}(i) Z_{1} y(i)+2 \xi_{0}^{T}(k) M_{1} \Lambda_{1} \\
&-\sum_{i=k-d_{M}}^{k-d(k)-1} y^{T}(i) Z_{1} y(i)+2 \xi_{0}^{T}(k) M_{2} \Lambda_{2} \\
&-\sum_{i=k-d_{M}}^{k-1} y^{T}(i) Z_{2} y(i)+2 \xi_{0}^{T}(k) M_{3} \Lambda_{3} \\
&-\sum_{i=1}^{+\infty} \mu_{i} f^{T}(x(k-i)) R f(x(k-i)) \\
&\left.+\bar{\mu} f^{T}(x(k)) R f(x(k))\right\},
\end{aligned}
$$

with $\Lambda_{1}=x(k)-x(k-d(k))-\sum_{i=k-d(k)}^{k-1} y(i), \Lambda_{2}=$ $x(k-d(k))-x\left(k-d_{M}\right)-\sum_{i=k-d_{M}}^{k-d(k)-1} y(i)$ and $\Lambda_{3}=$ $x(k)-x\left(k-d_{M}\right)-\sum_{i=k-d_{M}}^{k-1} y(i)$.

Notice that

$$
\begin{aligned}
& -\sum_{i=k-d(k)}^{k-1} y^{T}(i) Z_{1} y(i)-2 \xi_{0}^{T}(k) M_{1} \sum_{i=k-d(k)}^{k-1} y(i) \\
& \quad \leq d_{M} \xi_{0}^{T}(k) M_{1} Z_{1}^{-1} M_{1}^{T} \xi_{0}(k) .
\end{aligned}
$$

Similarly, we have

$$
\begin{aligned}
& -\sum_{i=k-d_{M}}^{k-d(k)-1} y^{T}(i) Z_{1} y(i)-2 \xi_{0}^{T}(k) M_{2} \sum_{i=k-d_{M}}^{k-d(k)-1} y(i) \\
& \quad \leq\left(d_{M}-d_{m}\right) \xi_{0}^{T}(k) M_{2} Z_{1}^{-1} M_{2}^{T} \xi_{0}(k)
\end{aligned}
$$

and

$$
\begin{aligned}
& -\sum_{i=k-d_{M}}^{k-1} y^{T}(i) Z_{2} y(i)-2 \xi_{0}^{T}(k) M_{3} \sum_{i=k-d_{M}}^{k-d(k)-1} y(i) \\
& \quad \leq d_{M} \xi_{0}^{T}(k) M_{3} Z_{2}^{-1} M_{3}^{T} \xi_{0}(k) .
\end{aligned}
$$

On the other hand, it follows from (7) and (12) that

$$
\begin{aligned}
& \sigma^{T}(x(k), x(k-d(k))) P \sigma(x(k), x(k-d(k))) \\
& \leq \lambda\left[x^{T}(k) \Sigma_{1}^{T} \Sigma_{1} x(k)\right. \\
&\left.+x^{T}(k-d(k)) \Sigma_{2}^{T} \Sigma_{2} x(k-d(k))\right] .
\end{aligned}
$$

Also, one has from Lemma 2 that

$$
\begin{gathered}
-\sum_{i=1}^{+\infty} \mu_{i} f^{T}(x(k-i)) R f(x(k-i)) \\
\leq-\frac{1}{\bar{\mu}}\left(\sum_{m=1}^{+\infty} \mu_{m} f(x(k-m))\right)^{T} \\
\times R \sum_{m=1}^{+\infty} \mu_{m} f(x(k-m)) .
\end{gathered}
$$

Furthermore, from (6) and (8), we have

$$
\left[\begin{array}{c}
x(k) \\
f(x(k))
\end{array}\right]^{T}\left[\begin{array}{cc}
\breve{L}_{1} & -\breve{L}_{2} \\
-\breve{L}_{2}^{T} & I
\end{array}\right]\left[\begin{array}{c}
x(k) \\
f(x(k))
\end{array}\right] \leq 0,
$$

and

$$
\begin{gathered}
g^{T}(x(k), x(k-d(k))) g(x(k), x(k-d(k))) \\
\leq-x(k-d(k)) G_{2}^{T} G_{2} x(k-d(k)) \\
\quad-x^{T}(k) G_{1}^{T} G_{1} x(k) .
\end{gathered}
$$

Letting

$$
\begin{aligned}
\Upsilon:= & \Psi_{0}+\eta_{0}^{T}\left(P+d_{M}\left(Z_{1}+Z_{2}\right)\right) \eta_{0}+\Xi_{1}+\Xi_{1}^{T} \\
& +\Xi_{2}+\Xi_{2}^{T}+\left(d_{M}-d_{m}\right) M_{2} Z_{1}^{-1} M_{2}^{T} \\
& +d_{M} M_{1} Z_{1}^{-1} M_{1}^{T}+d_{M} M_{3} Z_{2}^{-1} M_{3}^{T}
\end{aligned}
$$


and then substituting (28)-(34) into (27), we obtain that

$\mathbb{E}\{\Delta V(k)\} \leq \mathbb{E}\left\{\xi_{0}^{T}(k) \Upsilon \xi_{0}(k)\right\}$.

By (13), (35) and Schur Complement, we have $\Upsilon<0$ and therefore

$$
\mathbb{E}\{\Delta V(k)\} \leq-\lambda_{\min }(\Upsilon) \mathbb{E}|x(k)|^{2}
$$

where $\lambda_{\min }(\Upsilon)$ is the minimum eigenvalue of $\Upsilon$. It follows from the Lyapunov stability theory that the closed-loop system $\left(\Sigma_{c}\right)$ is globally asymptotically stable in the mean square.

In Theorem 1, the stability analysis problem is dealt with for the closed-loop system $\left(\Sigma_{c}\right)$ with a given feedback gain and a sufficient condition is derived, which depends both the delays $d_{m}$ and $d_{M}$, in the form of LMIs to guarantee the mean-square asymptotic stability of the closed-loop system $\left(\Sigma_{c}\right)$. In the following, two subsequent results are given in order to facilitate the control design procedure.

Corollary 1 Let $K$ be a given real constant matrix. The closed-loop system $\left(\Sigma_{c}\right)$ is globally asymptotically stable in the mean square sense if there exist three positive definite matrices $X, Q$ and $R$, and a positive constant scalar $\lambda$ such that the following LMIs hold:

$X>\lambda I$

$\Omega=\left[\begin{array}{ccccccc}\tilde{X} & 0 & X \breve{L}_{2} & 0 & 0 & X A_{K}^{T} & \tilde{W}_{1} \\ * & -Q & 0 & 0 & 0 & X B^{T} & \tilde{W}_{2} \\ * & * & \bar{\mu} R-I & 0 & 0 & 0 & 0 \\ * & * & * & -\frac{1}{\bar{\mu}} R & 0 & C^{T} & 0 \\ * & * & * & * & -I & I & 0 \\ * & * & * & * & * & -X & 0 \\ * & * & * & * & * & * & -\hat{W}\end{array}\right]<0$.

where $\tilde{X}=-X+\bar{d} Q, \bar{d}=d_{M}-d_{m}+1,, W_{1}=$ $\Sigma_{1}^{T} \Sigma_{1}+G_{1}^{T} G_{1}-\breve{L}_{1}, W_{2}=\Sigma_{2}^{T} \Sigma_{2}+G_{2}^{T} G_{2}, \tilde{W}_{1}=$ $\left[\begin{array}{ll}X W_{1} & 0\end{array}\right], \tilde{W}_{2}=\left[\begin{array}{ll}0 & X W_{2}\end{array}\right]$, and $\hat{W}=\operatorname{diag}\left\{W_{1}, W_{2}\right\}$.

Proof: Let $\bar{X}=\operatorname{diag}\left\{X^{-1}, X^{-1}, I, I, I, I, \operatorname{diag}\{I, I\}\right\}$ and

$$
\begin{aligned}
\Omega_{0} & =\bar{X} \Omega \bar{X} \\
& =\left[\begin{array}{ccccccc}
\Omega_{011} & 0 & \breve{L}_{2} & 0 & 0 & A_{K}^{T} & \breve{W}_{1} \\
* & \Omega_{022} & 0 & 0 & 0 & B^{T} & \breve{W}_{2} \\
* & * & \Omega_{033} & 0 & 0 & 0 & 0 \\
* & * & * & -\frac{1}{\bar{\mu}} R & 0 & C^{T} & 0 \\
* & * & * & * & -I & I & 0 \\
* & * & * & * & * & -X & 0 \\
* & * & * & * & * & * & -\hat{W}
\end{array}\right],
\end{aligned}
$$

with $\hat{W}$ has been defined in $(38)$ and $\Omega_{011}=-X^{-1}+$ $\bar{d} X^{-1} Q X^{-1}, \Omega_{022}=-X^{-1} Q X^{-1}, \Omega_{033}=\bar{\mu} R-I, \breve{W}_{1}=$ $\left[\begin{array}{ll}W_{1} & 0\end{array}\right], \breve{W}_{2}=\left[\begin{array}{ll}0 & W_{2}\end{array}\right]$. It is obvious that $\Omega<0$ is equivalent to $\Omega_{0}<0$. Furthermore, by letting $P=X^{-1}$ and $\hat{Q}=P Q P$, it follows readily from Schur complement that $\Omega_{0}<0$ is equivalent to

$$
\Omega_{2}:=\Omega_{1}+\eta^{T} P \eta<0 .
$$

where

$$
\Omega_{1}=\left[\begin{array}{ccccc}
\Omega_{111} & 0 & \breve{L}_{2} & 0 & 0 \\
* & -\hat{Q}+W_{2} & 0 & 0 & 0 \\
* & * & \bar{\mu} R-I & 0 & 0 \\
* & * & * & -\frac{1}{\bar{\mu}} R & 0 \\
* & * & * & * & -I
\end{array}\right]
$$

with $\Omega_{111}=-P+\bar{d} \hat{Q}+W_{1}$.

Construct the Lyapunov-Krasovskii functional $V(k)=$ $\sum_{i=1}^{4} V_{i}(k)$, where

$$
\begin{aligned}
& V_{1}(k)=x^{T}(k) P x(k), V_{2}(k)=\sum_{i=k-d(k)}^{k-1} x^{T}(i) \hat{Q} x(i), \\
& V_{3}(k)=\sum_{j=k-d_{M}+1}^{k-d_{m}} \sum_{i=j}^{k-1} x^{T}(i) \hat{Q} x(i), \\
& V_{4}(k)=\sum_{i=1}^{+\infty} \mu_{i} \sum_{j=k-i}^{k-1} f^{T}(x(j)) R f(x(j)) .
\end{aligned}
$$

The rest of the proof follows directly from Theorem 1 and is therefore omitted to save space.

Next, we are in a position to consider the stabilizability of the system $(\Sigma)$ and design the desired controller. The following result is given without proof since it is easily accessible from Corollary 1.

Corollary 2 System $(\Sigma)$ is stabilizable in the mean square sense if there exist three positive definite matrices $X, Q$ and $R$, a matrix $Y$, and a positive constant scalar $\lambda$ such that the following LMIs hold:

$X>\lambda I$

$\Omega=\left[\begin{array}{ccccccc}\tilde{X} & 0 & X \breve{L}_{2} & 0 & 0 & \tilde{A} & \tilde{W}_{1} \\ * & -Q & 0 & 0 & 0 & X B^{T} & \tilde{W}_{2} \\ * & * & \bar{\mu} R-I & 0 & 0 & 0 & 0 \\ * & * & * & -\frac{1}{\mu} R & 0 & C^{T} & 0 \\ * & * & * & * & -I & I & 0 \\ * & * & * & * & * & -X & 0 \\ * & * & * & * & * & * & -\hat{W}\end{array}\right]<0$

where $\tilde{X}, \tilde{W}_{1}, \tilde{W}_{2}, \hat{W}, W_{1}$ and $W_{2}$ are defined in Corollary 1 and $\tilde{A}=X A^{T}+Y^{T} D^{T}$. Furthermore, if LMIs (45)-(46) are feasible, the desired state feedback gain matrix can be designed by $K=Y X^{-1}$.

Remark 3 The features of the main results can be summarized as follows: 1) the distributed time-delay is defined in the discrete-time setting; 2) a new Lyapunov- 
Krasovskii functional is introduced to account for distributed time-delay; 3) a sector-like nonlinearity is imposed on the function concerning the distributed delays; and 4) an up-to-date delay-dependent approach is employed to obtain the LMI-based stabilizability conditions. We like to point out that, within the same LMI framework, it is not difficult to extend our main results to more general systems (e.g. parameter uncertain systems, systems with input delays and systems with uncertain switching probability) with static/dynamic output feedback.

\section{Numerical Example}

In this example, we consider the third-order system $(\Sigma)$ with the following parameters and nonlinear functions:

$$
\begin{aligned}
& A=\left[\begin{array}{ccc}
1 & 0.1 & 0 \\
0 & 0.3 & 0.1 \\
0.1 & 0 & -0.2
\end{array}\right], B=\left[\begin{array}{ccc}
0.2 & -0.1 & 0 \\
0.1 & -0.1 & 0 \\
0 & -0.2 & -0.1
\end{array}\right] \\
& C=\left[\begin{array}{ccc}
-0.2 & 0 & 0.1 \\
-0.2 & -0.1 & 0.1 \\
0 & 0.2 & -0.1
\end{array}\right], D=\left[\begin{array}{cc}
-1 & 1 \\
0 & 1 \\
0 & 1
\end{array}\right] \\
& d(k)=2+\frac{1+(-1)^{k}}{2}, \mu_{m}=2^{-(3+m)} \\
& f(x)=\left(f_{1}(x), f_{2}(x), f_{3}(x)\right)^{T} \\
& g(x, y)=\left(g_{1}(x, y), g_{2}(x, y), g_{3}(x, y)\right)^{T} \\
& \sigma(x, y)=g(x, y)
\end{aligned}
$$

where

$$
\begin{aligned}
& f_{1}(x)=\tanh \left(-x_{1}\right)+0.2 x_{1}+0.1 x_{2}+0.1 x_{3}, \\
& f_{2}(x)=0.1 x_{1}-\tanh \left(x_{2}\right)+0.2 x_{2}, \\
& f_{3}(x)=0.1 x_{1}+0.2 x_{3}-\tanh \left(x_{3}\right), \\
& g_{1}(x, y)=-0.2 \sqrt{x_{1}^{2}+y_{2}^{2}} \sin \left(x_{1}^{2}+x_{2}^{2}\right), \\
& g_{2}(x, y)=0.2 \sqrt{x_{2}^{2}+y_{1}^{2}} \cos \left(x_{1}^{2}+x_{2}^{2}\right), \\
& g_{3}(x, y)=0.2 \sqrt{x_{3}^{2}+y_{3}^{2}} .
\end{aligned}
$$

It is easy to verify that

$$
\begin{aligned}
L_{1} & =\left[\begin{array}{ccc}
-0.8 & 0.1 & 0.1 \\
0.1 & -0.8 & 0 \\
0.1 & 0 & -0.8
\end{array}\right], L_{2}=\left[\begin{array}{ccc}
0.2 & 0.1 & 0.1 \\
0.1 & 0.2 & 0 \\
0.1 & 0 & 0.2
\end{array}\right], \\
\Sigma_{1} & =\Sigma_{2}=G_{1}=G_{2}=0.2 I, \\
d_{m} & =2, d_{M}=3, \bar{\mu}=2^{-3} .
\end{aligned}
$$

With the above parameters, by using Matlab LMI Toolbox, we solve the LMIs (45)-(46) and obtain the desired feedback gain matrix as follows

$$
K=Y X^{-1}=\left[\begin{array}{ccc}
0.9628 & -0.0883 & 0.0116 \\
-0.0461 & -0.1618 & 0.0382
\end{array}\right] \text {. }
$$

According to Corollary 2, the system $(\Sigma)$ with the given parameters is stabilizable in the mean square and such a conclusion is further confirmed by the numerical simulation. In fact, Fig. 1 shows the dynamics evolution of the uncontrolled system $(\Sigma)$, i.e., in the case of $u(k) \equiv 0$. In this case, it is observed that the system is unstable. As shown in Fig. 2, the closed-loop system with the above feedback gain matrix is stable. Therefore, the simulation matches the theoretical results perfectly.
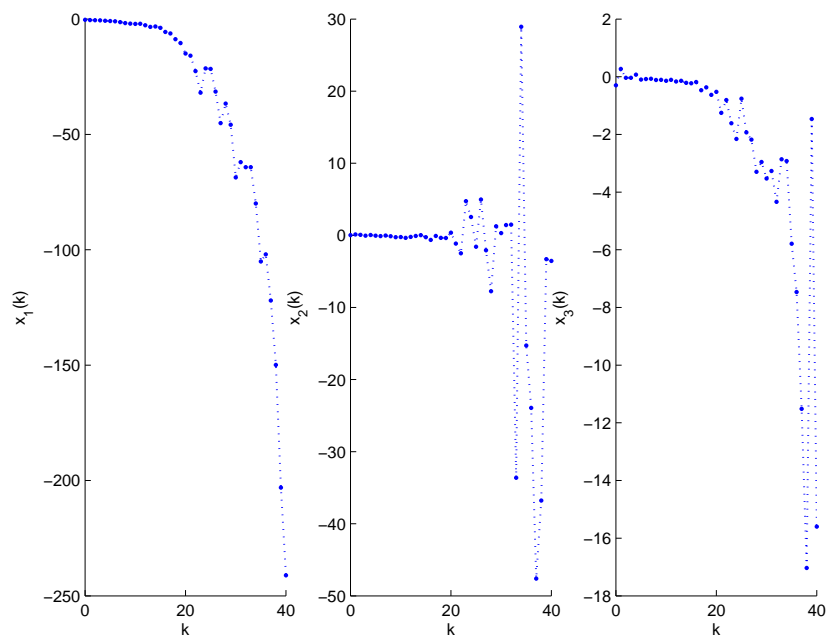

Fig. 1. The state evolution of the uncontrolled system
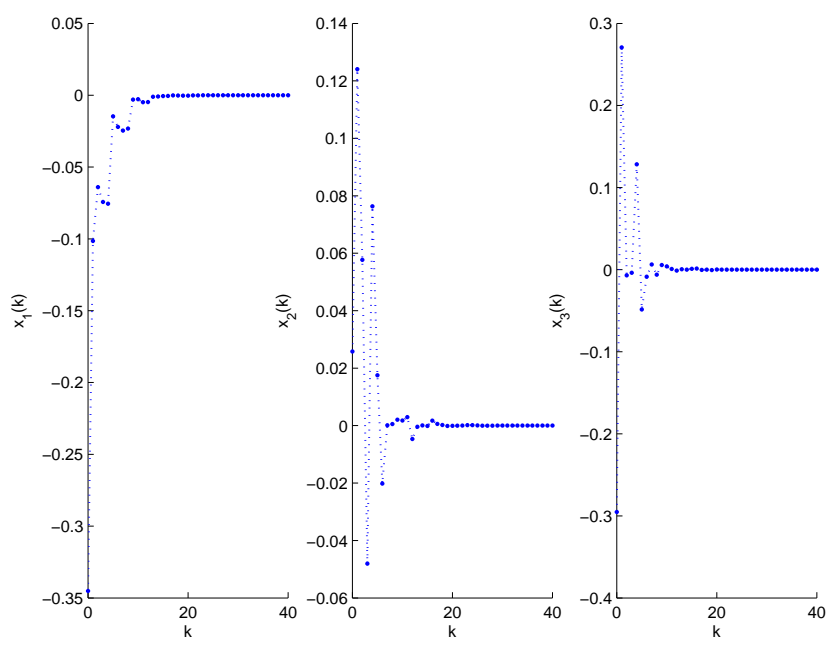

Fig. 2. The state evolution of the closed-loop system

\section{References}

[1] E. K. Boukas and Z. -K. Liu, Deterministic and stochastic time-delay systems, Birkhauser, Boston, 2002.

[2] N. Berman and U. Shaked, $H_{\infty}$ control for discrete-time nonlinear stochastic systems, IEEE Trans. Automat. Control, vol. 51, no. 6, pp. 1041-1046, 2006.

[3] E. Fridman and G. Tsodik, $H_{\infty}$ control of distributed and discrete delay systems via discretized Lyapunov functional, European Journal of Control, vol. 15, no. 1, pp. 84-96, 2009.

[4] E. Fridman and Y. Orlov. Exponential stability of linear distributed parameter systems with time-varying delays, Automatica, vol. 45, no. 2, pp. 194-201, 2009.

[5] H. Gao, J. Lam and C. Wang, Robust energy-to-peak filter design for stochastic time-delay systems, Systems $\&$ Control Letters, vol. 55, no. 2, pp. 101-111, 2006. 
[6] H. Gao and T. Chen, New results on stability of discrete-time systems with time-varying state delay, IEEE Trans. Automat. Control, vol. 52, no. 2, pp. 328-334, 2007.

[7] Q.-L. Han, Absolute stability of time-delay systems with sector-bounded nonlinearity, Automatica, vol. 41, no. 12, pp. 2171-2176, 2005.

[8] D. W. C. Ho, Robust fuzzy design for nonlinear uncertain stochastic systems via sliding-mode control, IEEE Trans. Fuzzy Systems, vol. 15, no. 3, pp. 350-358, 2007.

[9] H. K. Khalil, Nonlinear systems. Upper Saddle River, NJ: Prentice-Hall, 1996.

[10] V. Kolmanovskii and A. D. Myshkis, Introduction to the theory and applications of functional differential equations. Kluwer Academic Publishers, Boston, 1999.

[11] V. Kolmanovskii and L. Shaikhet, About one application of the general method of Lyapunov functionals construction, Int. J. Robust and Nonlinear Control, vol. 13, no. 9, pp. 805818, 2003.

[12] J. Lam, H. Gao, S. Xu and C. Wang, $H_{\infty}$ and $L_{2} / L_{\infty}$ model reduction for system input with sector nonlinearities, J. Optimization Theory and Applications, vol. 125, no. 1, pp. 137-155, 2005.

[13] Y. Liu, Z. Wang, J. Liang and X. Liu, Synchronization and state estimation for discrete-time complex networks with distributed delays, IEEE Trans. Syst. Man Cybern.-Part B, vol. 38, no. 5, pp. 1314-1325, Oct. 2008.

[14] W. M. McEneaney, G. Yin and Q. Zhang (Eds.), Stochastic analysis, control, optimization and applications, Systems and Control: Foundations and Applications series, Birkhauser Boston, Cambridge MA, 1999.

[15] S.-I. Niculescu. Delay effects on stability: a robust control approach, vol. 269 of Lecture Notes in Control and Information Sciences. Springer-Verlag, London, 2001.

[16] Y. Niu and D. W. C. Ho, Design of sliding mode control for nonlinear stochastic systems subject to actuator nonlinearity, IEE Proc. Control Theory and Applications, vol. 153, no. 6, pp. 737-744, 2006.

[17] L. Shaikhet, Stability of systems of stochastic linear difference equations with varying delays, Theory of Stoch. Process, vol. 4(20), no. 1-2, pp. 258-273, 1998.

[18] Z. Wang, D. W. C. Ho and X. Liu, A note on the robust stability of uncertain stochastic fuzzy systems with timedelays, IEEE Trans. Systems, Man and Cybernetics - Part $A$, vol. 34, no. 4, pp. 570-576, 2004.

[19] W. M. Wonham and A. B. Reid, Ed., Random differential equations in control theory, in Probabilistic Methods in Applied Mathematics. New York: Academic, 1970, vol. 2.

[20] L. Xie, E. Fridman and U. Shaked, Robust $H_{\infty}$ control of distributed delay systems with application to combustion control, IEEE Trans. Automat. Control, vol. 46, no. 12, pp. 1930-1935, 2001. 